\title{
Acessibilidade em redes sociais: em busca da inclusão digital no Facebook
}

\author{
Accessibility in social networks: \\ in search of digital inclusion in Facebook
}

\begin{abstract}
Resumo: A utilização de redes sociais cresce a cada dia. Usuários fazem uso das redes sociais para as mais diversas finalidades e estas representam uma forma de interação entre indivíduos, bem como trazem consigo uma série de informações e dados que podem ser utilizados para o desenvolvimento pessoal. Indivíduos que apresentam determinadas deficiência podem vir a ter dificuldade de acesso, pois algumas redes sociais não estão de acordo com as normas de acessibilidade. Neste sentido este artigo tem como objetivo realizar uma analise da acessibilidade das redes sociais através do uso de ferramentas, bem como propor algumas mudanças que possam vir a facilitar o acesso pelos indivíduos com deficiência.

Palavras-chave: Redes Sociais. Zona de Desenvolvimento Proximal. Acessibilidade
\end{abstract}

\begin{abstract}
The use of social networks is growing every day. Users make use of social networks for many different purposes and these represent a form of interaction between individuals, and bring with them a wealth of information and data that can be used for personal development. Individuals with certain disabilities can be expected to have limited access, as some social networks are not in accordance with accessibility standards. In this sense this article aims to conduct an analysis of the accessibility of social networking through the use of tools, and propose some changes that may facilitate access by individuals with disabilities.

Keywords: Social Networks. Zone of Proximal Development. Accessibility
\end{abstract}

WAGNER, Rosana; PIOVESAN, Sandra; RODRIGUES, Lisete. Acessibilidade em redes sociais: em busca da inclusão digital no Facebook. Informática na Educação: teoria e prática, Porto Alegre, v. 16, n. 2, p. 51-61, jul./dez. 2013.

\section{Rosana Wagner}

Sandra Piovesan

Lisete Rodrigues

Universidade Federal do Rio Grande do Sul

\section{Introdução}

A mudança tecnológica implica profundas alterações na compreensão dos processos de interação social e na construção da aprendizagem e do conhecimento (BARROS, 2011). A comunicação, colaboração/cooperação e interação que são possibilitadas através da WEB 2.0 tornam-se um espaço de fundamental importância para o desenvolvimento de qualquer individuo. Pessoas com deficiências tendem a sofrer a infoexclusão pelas suas dificuldades de acesso e problemas de acessibilidade nos ambientes atuais.

Desta forma as redes sociais são fundamentais para o desenvolvimento pessoal e social já que permitem a interação entre pessoas conhecidas bem como o conhecimento de novas pessoas e o início de novos círculos de 
convivência. E, passam a ser então uma forma de promover a educação para o pensar, abordando novas formas de aprendizagem em ambientes colaborativos/cooperativos.

A partir dos conceitos definidos anteriormente tem-se por objetivo o desenvolvimento de uma rede social acessível e personalizável, que possibilite a interação entre as pessoas com necessidades especiais já estudadas pelos grupos de pesquisa integrantes do NIEE (Núcleo de Informática na Educação Especial) UFRGS. Este artigo é parte inicial integrante de um trabalho e visa encontrar os principais problemas relacionados a acessibilidade web, para que estas dificuldades possam ser previstas no momento da criação da rede social acessível.

$\mathrm{Na}$ educação isso pode representar a oportunidade de grandes transformações e segundo Paloff (2002, p. 17), "fazer a transição da sala de aula para o ciberespaço e compreender mais completamente as novas abordagens e habilidades que precisam para ter sucesso". Isso porque, ações pedagógicas baseadas nesta tecnologia podem permitir que os alunos criem a cultura de produzir conteúdos e de debatê-los entre os membros de uma rede social, por exemplo, ganhando definitivamente uma voz mais ativa e participativa nos processos de ensino-aprendizagem, além de possibilitar a inclusão de todos.

Nesse sentido, a rede social escolhida - o Facebook - deve ser visto como ferramenta a disposição do professor para promover a acessibilidade.

Este artigo está organizado como segue: nos itens 2 e 3 são apresentadas as revisão bibliográficas sobre redes sociais e acessibilidade respectivamente. $\mathrm{O}$ item 4 apresenta a relação entre a teoria de Vigotsky com as redes sociais. No item 5 é apresentada a metodologia utilizada para a avaliação das redes sociais. No item 6 são apresentados os principais problemas encontrados e são propostas algumas meIhorias. 0 item 7 apresentada as conclusões do trabalho. No item 8 apresentam-se os agradecimentos e finalmente no item 9 são apresentadas as referencias bibliográficas utilizadas.

\section{Redes sociais}

As redes sociais apresentam um número de participantes e formas de utilização que aumentam diariamente, nomeadamente para interagir com pessoas conhecidas ou para conhecer novas pessoas (MORO 2001). A interação, transmissão e partilha de informações entre membros de grupos de interesse comum é um dos principais motivadores da ampla utilização das redes sociais. As redes sociais dão vida a seus autores, possibilitando que o mesmo configure seu perfil de acordo com seu interesse e contendo as informações que gostaria de divulgar, possibilitando assim a exposição do sujeito de acordo com seus interesses.

O sentido da construção coletiva e colaborativa na Web possibilita a construção do conhecimento em grupo e a troca de informações. Porém, este processo estende-se para além da perspectiva tecnológica da mediação e incide igualmente, de forma mais particular, nas práticas de mediação social e cognitiva entre os membros que interagem na rede, transformando o conjunto destas narrativas coletivas e na experiência de conhecimento partilhada pela comunidade (BARROS, 2011).

As redes sociais têm vindo a assumir um papel cada vez mais central na Web 2.0, a qual visa centrar a Web como uma plataforma que aproveita o efeito de rede, tendo em vista que quanto mais as aplicações forem utilizadas mais ricas se tornam. 
Segundo Recuero (2009), entre as redes sociais mais utilizadas atualmente está o Facebook, o Orkut, o Twitter, o LinkedIn, o Ebah, o MySpace e o hi5 . Cada uma destas tem um enfoque diferenciado, como, por exemplo, o Ebah é focado em compartilhamento de arquivos como livros e artigos e o LinkedIn é focado na troca de informações profissionais.

\section{Acessibilidade}

Os recursos disponíveis na Web são a cada dia mais interessantes, auto-explicativos e dinâmicos. São ferramentas que vem com o intuito de disseminar o uso da Web ainda mais, tornando sites e redes sociais atrativos, interessantes e divertidos. Mas estas representações gráficas têm sido, na maioria das vezes, fatores excludentes para um número significativo de sujeitos com necessidades especiais (SANTA ROSA et al. 2010). Estes fatores impossibilitam que as pessoas com deficiências participem destes ambientes, ou ao menos diminuem suas possibilidades de interações.

Desta forma, faz-se necessário para a construção de conteúdo acessível, a consonância com modelos e regras de acessibilidade no desenvolvimento de sites, redes sociais, ambientes virtuais, etc.

O W3C (World Wide Web Consortium) buscando garantir a utilização de padrões na Web publicou, em maio de 1999, as Diretrizes para Acessibilidade do Conteúdo Web 1.0 (Web Content Accessibility Guidelines - WCAG 1.0), que atualmente está em sua versão 2.0 - (W3C, 2012), sendo, até hoje, as principais referências em torno de acessibilidade a web no mundo.

Este documento está organizado em quatro princípios norteadores que possibilitam o acesso, a qualquer pessoa, ao conteúdo da web (W3C, 2012): Princípio da percepção: as informações e os conteúdos deve ser perceptíveis ao usuário; Princípio da operação: tornar os elementos de interface com o usuário operáveis; Princípio da compreensão: Conteúdos e controles devem ser compreensíveis ao usuário; Princípio da robustez: o conteúdo deve ser robusto o suficiente para trabalhar com tecnologias atuais e futuras.

\section{Redes sociais relacionadas à teo- ria de Vigotski}

$\mathrm{Na}$ teoria sócio-interacionista, proposta por Vigotsky, o foco de suas preocupações foi o desenvolvimento do sujeito e da espécie humana, como resultado de um processo sócio-histórico, por entender que o ser humano é o resultado das determinações de sua estrutura biológica e, principalmente de sua conjuntura histórica, pois as características humanas não estão presentes desde o nascimento do indivíduo, nem são mero resultados das pressões do meio externo. Pelo contrário, elas resultam da interação dialética do ser humano e o seu meio sócio-cultural, ou seja, quando o ser humano modifica o ambiente através de seu próprio comportamento, essa mesma modificação vai influenciar seu comportamento futuro.

A partir de uma perspectiva em que a socialização tem uma função determinante no desenvolvimento humano, Vigotsky buscou identificar de que forma as características tipicamente humanas que ele chamou de Funções Psicológicas Superiores (FPS), se desenvolvem durante a vida de um indivíduo. As teorizações, as quais Vigotsky e seus colaboradores chegaram, servem como base para se pensar e propor uma educação inclusiva, através do uso 
das Redes Sociais Acessíveis como ambientes de aprendizagem cooperativa, que permitam a inclusão de alunos com deficiência.

Em consonância com esta forma de utilização das Redes Sociais Acessíveis, está o conceito de Zona de Desenvolvimento Proximal, proposto por Vygotsky (2007), que é a distância entre o nível de desenvolvimento real, que se costuma definir através da solução independente de problemas, e o nível de desenvolvimento potencial, determinado através da solução de problemas sob a orientação de um adulto ou em colaboração com companheiros mais capazes. Além disso, a interação entre indivíduos atende a perspectiva construtivista/ interacionista, pois as redes sociais, possibilitam a interação com companheiros mais capazes e resultam na formação de um sujeito autônomo e ativo na estruturação de seu conhecimento. Vygotsky 2007, influenciado pelo materialismo histórico dialético de Marx concebe que o organismo ativo interage com um ambiente histórico e essencialmente social e a partir disso constrói seu processo de desenvolvimento.

As redes sociais podem ser vistas como formas de aplicação dos princípios da abordagem sócio-interacionista proposta por Vygotsky, o qual buscava investigar de que forma a interação social promove o desenvolvimento de Funções Psicológicas Superiores no decorrer da vida de um indivíduo (VYGOTSKY, 2007). Segundo o autor, as crianças crescem imersas em um ambiente de constante interação com os adultos, e oferecem ao mundo, inicialmente, respostas dominadas pelos processos naturais. Porém, através da constante mediação dos adultos, processos instrumentais mais complexos começam a tomar forma. Em um primeiro momento, estes processos só podem funcionar durante a interação das crianças com os adultos (processos interpsíquicos), os quais servem de mediadores do contato da criança com o mundo. No decorrer do crescimento, estes processos acabam por ser executados dentro das próprias crianças. Isto é, as respostas mediadoras ao mundo transformam-se em um processo intrapsíquico (VIGOTSKI; LURIA; LEONTIEV, 2006, p. 27). A este processo de apropriação Vygotsky dá o nome de internalização, e destaca também, como parte do mesmo, a reconstrução de operações representantes de atividades externas de forma que as mesmas ocorram internamente (VYGOTSKY, 2007).

Traduzindo para a atualidade trechos descritos por Vygotsky, pode-se notar a real importância da utilização de ambientes tecnológicos no desenvolvimento cognitivo. Engels e Vygotsky criticam os psicólogos e filósofos que sustentavam "que apenas a natureza afeta o homem e apenas as condições naturais determinam o desenvolvimento histórico do homem, enfatizando que ao longo da historia o homem também "afetam a natureza", transformando-a, criando para si novas condições naturais de existência." Além disso, Vygotsky argumentou que o efeito do uso de instrumentos sobre os homens é fundamental não apenas porque os ajuda a se relacionarem mais eficazmente com seu ambiente como também devido aos importantes efeitos que o uso de instrumentos tem sobre as relações internas e funcionais no interior do cérebro humano (VYGOTSKY 1991). As redes sociais foram desenvolvidas pelos homens e são utilizadas como instrumento facilitador e que contribui para a socialização dos indivíduos. Porém há divergência na capacidade de realizar acesso. Algumas pessoas têm mais facilidade outras menos. As pessoas com necessidades especiais devem conseguir realizar acesso da mesma maneira que as demais, por isso a importância da acessibilidade. 
A acessibilidade pode ser classificada como um instrumento facilitador desenvolvido pelo homem para garantir o acesso a todas as pessoas.

No sentido de auxiliar o desenvolvimento cognitivo de crianças com necessidades especiais, fazendo com que a ferramenta seja uma forma de inclusão de pessoas com deficiência, tem-se também apoio da teoria de Vygotsky 1997, que afirma que crianças retardadas mentais $^{1}$ não são muito capazes de terem pensamento abstrato, então seria mais fácil simplesmente basear-se no uso de métodos do tipo "observar e fazer". Porém mesmo com a criança tendo dificuldade é necessário que se estimule ao pensar abstrato, possibilitando assim algum desenvolvimento em relação ao abstrato e não mantendo-a somente no concreto. O interessante é estimular para desenvolver algumas possibilidades do pensar abstrato na criança. Esta teoria tem relação com a utilização de redes sociais acessíveis, através do qual a criança poderá utilizar-se tanto do concreto quanto do abstrato, através de conversas e interações com outros indivíduos.

\section{Validação para acessibilidade web}

Segundo a UNESCO, desde os anos 80, vem crescendo o entendimento de que as dificuldades impostas pelos limites de uma deficiência a um indivíduo variam segundo a cultura e desenvolvimento tecnológico de cada país ou região. Portanto não é apenas o tipo e o grau de deficiência sensorial, cognitiva ou física que determina a limitação de uma pessoa; o ambiente no qual ela se insere também pode fazer com que fique mais ou menos limitada.

\footnotetext{
O termo foi mantido, conforme o original.
}

Aplicando-se essa ideia às páginas da Web, podemos entender como a tecnologia tanto pode, quando bem utilizada, contribuir para maior qualidade de vida para inúmeras pessoas, como pode vir a se constituir, se for mal empregada, numa grande fonte de frustração.

Segundo Conforto e Santarosa (2002), "são as ferramentas digitais que, ao atuarem como objetos catalisadores da inteligência coletiva, tornam-se entidades que propiciam o acolhimento da diversidade. As redes digitais planetárias, especialmente as ferramentas da Internet, abrem um enorme campo de possibilidades para o lazer, para a formação, para o trabalho e para a vida social, potencializando a inclusão e a valorização da diversidade humana" (CONFORTO; SANTAROSA, 2002).

Esta temática - Acessibilidade à Web - no que se refere ao planejamento, à revisão, à avaliação e à validação de páginas WEB, traz para a discussão a necessidade e a importância da construção de conteúdo acessível para a Web como facilitadora da inserção da diversidade humana no ciberespaço.

Segundo Santarosa, et al. 2010, a avaliação da acessibilidade de uma página web deve estar presentes desde as fases iniciais de desenvolvimento do conteúdo para a internet. Para proceder a avaliação e validação da acessibilidade de sites temos os avaliadores automáticos - ferramenta para o uso de desenvolvedores de sites para a Internet, em consonância com os princípios de acessibilidade do W3C. Ainda referenciando Santarosa et al. 2010, a avaliação da acessibilidade deve ser feita por meio destas ferramentas automáticas, mas também através da revisão manual. Os métodos automáticos são geralmente rápidos, mas não são capazes de identificar todos os aspectos da acessibilidade. A avaliação humana pode ajudar a garantir a clareza da linguagem e a facili- 
dade de navegação (SANTAROSA, et al. 2010). Para esta finalidade o grupo do NIEE/UFRGS criou uma Ficha de Apoio à Avaliação Manual de Acessibilidade à Web na qual são observados e avaliados 17 itens a serem cumpridos para a adequação de um site às Diretrizes e aos Princípios de Acessibilidade à Web.

\subsection{Validação automática}

Algumas ferramentas que auxiliam na avaliação são:

Da Silva - O Da Silva é um software avaliador de acessibilidade, brasileiro, que detecta um código HTML e faz uma análise do seu conteúdo, verificando se está ou não dentro do conjunto de regras do WCAG e E-GOV, preconizados pela lei federal de acessibilidade (Lei no. 10 098, de 19 de dezembro de 2000), que estabelece normas gerais e critérios básicos para a promoção da acessibilidade das pessoas portadoras de deficiência ou com mobilidade reduzida e dá outras providências.

WebXACT - É um serviço gratuito on-line (em substituição ao antigo Bobby) que permite testar páginas Web simples.

Total validator - O Total validator é a ferramenta que faz a avaliação da linguagem HTML, CSS, links quebrados e Acessibilidade, segundo os padrões W3C WAI WCAG 1.0 e WCAG 2.0 e Section 508 (Administração de Serviços Gerais de Política Governamentabilidade dos EUA - tecnologia eletrônica e da informação acessível a pessoas com deficiência nos EUA). É uma das poucas ferramentas de avaliação que também avalia a semântica das páginas.

Hera - é uma ferramenta para rever a acessibilidade das páginas Web de acordo com as recomendações das Diretrizes de Acessibilidade para o Conteúdo Web 1.0 (WCAG 1.0).
O Hera efetua uma análise automática prévia da página e disponibiliza informação dos erros encontrados (detectáveis de forma automática) e quais os pontos de verificação que devem ser revistos manualmente. O hera facilita a revisão manual, proporcionando informação sobre os elementos a verificar, instruções de como realizar esse controle e duas visões modificadas da página (uma visão gráfica, outra do código HTML) com os elementos mais importantes destacados com ícones e cores diferenciadoras. Disponibiliza também um formulário que permite modificar os resultados automáticos, agregar comentários a cada um dos pontos de verificação e indicar o nome do revisor.

TAW - Test Acessibilidade Web - uma família de ferramentas para a análise da acessibilidade de sites web, alcançando de forma integral e global todos os elementos e páginas que o compõem. Esta família é composta por diversas ferramentas, desde os mais conhecidos analisadores de páginas de sistemas motorizados ou uma ferramenta para a realização de observações. Tem como objetivo comprova o nível de acessibilidade no layout de páginas web com a finalidade de permitir o acesso a todas as pessoas independentes de suas características diferenciadoras. É destinada ao publico em geral e especificamente profissionais de campo como Webmasters.

W3C Validator - é um avaliador desenvolvido e mantido pela W3C, para fins de validação de acessibilidade de páginas Web.

Todas as ferramentas citadas têm características em comum, a avaliação de acessibilidade através dos princípios da WCAG. 


\subsection{Validação manual}

A validação manual é feita através da Ficha de Apoio à Avaliação Manual da Acessibilidade Web preconizada por Santarosa et al. 2010. Para a verificação da aplicação dos princípios de acessibilidade de um site, nesta ficha, são observados 17 itens considerados importantes para a adequação de um site às Diretrizes e aos Princípios de Acessibilidade à Web.

A revisão manual é imprescindível para comprovar realmente se a página é acessível. Para poder levar a cabo esta verificação manual é necessário conhecer as diretrizes de acessibilidade, saber de que forma fazem, os utilizadores, uso das ajudas técnicas e ter alguma experiência em desenho e desenvolvimento de páginas Web (Hera).

\subsection{Metodologia para avaliação da acessibilidade de redes sociais}

Optou-se pela avaliação da rede social mais utilizada atualmente, o Facebook.

A avaliação foi realizada através da utilização de sites avaliadores automáticos e da ficha de apoio a avaliação manual, pois conforme preconizado por Santarosa et al. 2010, as duas formas devem ser utilizadas, pois se completam.

A avaliação automática foi realizada, inicialmente pelas ferramentas Hera, Taw e Da Silva, conforme apresentado nas Figuras 1, 2 e 3, e posteriormente utilizou-se a ficha de apoio à avaliação manual da web por nós e também optamos por utilizar a avaliação de um "usuário real" cego, que integra a equipe de tutores do Curso de Formação Continuada de Professores em Tecnologias da Informação e Comunicação Acessível idealizado e ofertado pelo
NIEE/UFRGS em parceria com a Universidade Aberta do Brasil.

A rede social escolhida, o Facebook, foi avaliada inicialmente através das três ferramentas. Os resultados estão apresentados nas imagens abaixo.

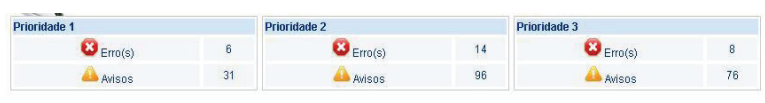

Figura 1. Avaliação do Facebook pela ferramenta DaSilva segundo as regras WCAG

Test summary outcome

\begin{tabular}{ccc} 
& & Automatic Human review \\
Priority 1 & 1 $>0$ & ?ㄴ 81 \\
Priority 2 & 2) 13 & 의 108 \\
Priority 3 & \multicolumn{2}{c}{ Not analysed }
\end{tabular}

Figura 2. Avaliação do Facebook pela ferramenta TAW

\begin{tabular}{|c|c|c|c|c|}
\hline Prioridade & Verificar & Bem & Mal & N/A \\
\hline \begin{tabular}{|c|c|} 
P1 & P1 \\
HIRAA WCAG 1.0
\end{tabular} & $7 \circ$ & -- & $1 \times$ & 9 \\
\hline $\begin{array}{|cc|}\text { P2 } & \text { P2 } \\
\text { HERAA WCAG 1.0 }\end{array}$ & 15 & $5 v$ & $4 \times$ & 5 \\
\hline $\begin{array}{|cc|}\times & \mathbf{P 3} \\
\text { HEl } & \text { WCAG } 1.0 \\
\end{array}$ & $11 \circ$ & $2 v$ & $1 \times$ & 5 \\
\hline
\end{tabular}

Figura 3. Avaliação do Facebook pela ferramenta Hera

Através da analise da avaliação realizada entre as três ferramentas utilizadas, nota-se que as prioridades de nível mais baixo - Prioridade 1 - correspondem a situações que devem necessariamente atender a normas de acessibilidade ou causarão danos a pessoas com deficiência que tem a intenção de utilizá-las. Prioridades médias - Prioridade 2 - correspondem a questões que seria importante serem implementadas para que as normas de acessibilidade fossem atendidas. Prioridades altas - Prioridade 3 - correspondem a ações que facilitariam o acesso caso implementadas.

Desta forma, é possível verificar que uma das ferramentas não acusou nenhum proble- 
ma em relação a prioridade 1 e as outras duas acusaram poucos problemas.

Posteriormente foi realizada pelo "usuário real" cego, a avaliação do site do FACEBOOK, utilizando o navegador Mozilla Firefox e o leitor de telas NVDA, no período de 24/11 a 01/12/2012. Foi avaliada apenas a interface do site, o conteúdo disponibilizado por sites integrados e seus usuários não entrou nesta avaliação.

Em relação a avaliação manual, realizada pelo usuário com deficiência visual, constata-se que dos 17 critérios analisados através da ficha de avaliação manual $\mathbf{2}$ são atendidas, $\mathbf{5}$ não são atendias e $\mathbf{1 0}$ não foram observadas.

É possível concluir que a rede social aqui analisada corresponde parcialmente às normas de acessibilidades, vista a forma, tamanho e quantidade de utilização da mesma.

\section{Problemas de acessibilidade em redes sociais e sugestões de mel- horias}

O site oficial http://www.facebook.com.br não oferece acessibilidade satisfatória a leitores de tela. Possui expressões em inglês, sem a tradução para português. Algumas funcionalidades não funcionam da forma apresentada, como a execução de músicas. O usuário precisa explorar o ambiente e consultar a ajuda, não existe um mapa descritivo das páginas que o compõem.

Esta falta de acessibilidade é reconhecida por seus construtores, pois no menu <ajuda> do site, é recomendado aos usuários de leitores de tela o acesso pela página destinada aos dispositivos móveis, construída em <HTM > simplificado. Entretanto não é oferecido o link direto para esta alternativa, somente é informado na ajuda.

Tanto na opção de acesso pelo site oficial, quanto na página destinada ao acesso através de dispositivos móveis foram encontrados links quebrados, como o link para confirmar solicitações e realizar leituras de mensagens.

A página destinada ao acesso através de dispositivos móveis http://m.facebook.com oferece boa acessibilidade a Tecnologia Assistiva leitores de tela. Neste, várias funcionalidades foram suprimidas, bem como a poluição dos anúncios patrocinados. A interface mais limpa favorece uma navegação mais eficiente para usuários com deficiência visual ou cegos.

Inicialmente, a sugestão do usuário cego consultado, para viabilizar maior acessibilidade, seria disponibilizar um mapa descritivo das páginas que compõe o site, oferecendo uma noção geral ao usuário de leitor de telas.

Os principais softwares de leitura de páginas web, para deficientes visuais, utilizam o campo de descrição alternativa (Alt), para explicar sobre do que se trata a imagem. Se esse campo estiver em branco, a imagem não será interpretada por esses softwares e não será devidamente transmitida para esse público. Em relação a isso, outro problema encontrado na rede social Facebook é relacionado à falta de textos alternativos indexados às imagens. Este descuido deixou algumas imagens, sem texto indexado, como do "menu ajuda". Neste caso é verbalizado apenas o número correspondente ao arquivo, pelo leitor de tela.

No menu <ajuda> do site é informada a possibilidade de navegar com atalhos do teclado, ou seja, chaves de acesso, para usuários com mobilidade reduzida e que fazem uso do computador utilizando apenas o teclado, o que facilita para pessoas que tem limitações motoras. 
O site apresenta maneiras de ajustar o zoom ou o tamanho da Tela: Pressionando a Tecla Ctrl ou Comando e pressionando a Tecla Menos (-) par reduzir o tamanho ou então segurando a Tecla Ctrl ou Cmd e pressionando a Tecla Mais (+) para Aumentar o tamanho atendendo as necessidades das pessoas com limitações visuais.

Outra forma de melhoria e de abrangência da acessibilidade é a inclusão de chats com vídeos, onde é possível realizar uma interação visual e auditiva, facilitando a integração das pessoas cegas e surdas. Há também a possibilidade de que os chats possam ser gravados e posteriormente, legendados ou traduzidos para a LIBRAS e disponibilizados, com a finalidade de atender às pessoas com limitações auditivas.

O site também possibilita tornar acessíveis as fotos postadas pelos usuários. A melhor maneira de garantir que fotos sejam acessíveis a todos é através da inclusão de legendas. Estas permitem descrever brevemente uma foto com o objetivo de garantir que pessoas com deficiência tenham uma descrição de texto, alternativo a imagem. Nesta opção vemos que é fundamental o trabalho cooperativo dos usuários do espaço de aprendizagem inclusivo que pode vir a ser criado no Facebook na opção "criar grupos", para atender aos critérios de acessibilidade. Dessa forma, é fundamental a conscientização das pessoas, no sentido de cooperar e transformar esta rede social em um ambiente inclusivo, descrevendo as imagens publicadas em suas postagens, bem como a necessidade de escrita ortográfica correta, evitando gírias e abreviações que os "leitores de páginas" não conseguem ler.

\section{Conclusão}

Destaca-se a relevância do tema da acessibilidade no contexto do planejamento e desenvolvimento e execução de sites em geral, especialmente nas redes sociais, considerando-se especificamente o Facebook, objeto deste estudo, para atender as limitações dos seus usuários, sejam elas cognitivas, motoras, visuais, ou auditivas.

O Facebook apoiado na teoria de Vigotsky permite a compreensão do processo ensino-aprendizagem, sob a perspectiva da mediação, pois a partir do estímulo e interação entre o indivíduo e o social, o impacto dos conhecimentos apreendidos a partir das interações dentro de um grupo criado na rede social, pode desenvolver a autonomia no pensamento crítico dos sujeitos envolvidos no processo ensino-aprendizagem, em que cada membro é responsável pela sua aprendizagem e pela aprendizagem dos outros participantes.

Conclui-se que muitas das alterações necessárias nesta rede social a fim de solucionar suas falhas críticas tornando-a mais acessível são, em sua maioria, simples de serem realizadas, conforme detalhado anteriormente. Mesmo com estas falhas, a maioria dos usuários com alguma limitação consegue acessar o Facebook, porém com dificuldade em determinados itens.

Sobre a questão da produção de um conteúdo acessível, no caso, um grupo no Facebook que funcione como um Ambiente Virtual de Aprendizagem, é necessário que se invista na conscientização e educação para a Acessibilidade à Web. Este é o único caminho, já que a ferramenta em si não poderá controlar o conteúdo gerado. 
Neste sentido o uso do Facebook como AVA, ou seja, como "instrumento" na perspectiva vigotskyana, funciona como um condutor da influência humana sobre o objeto da atividade é orientado externamente e leva necessariamente a mudanças no objeto, no caso em análise, buscando através das melhorias na acessibilidade do site, a universalidade da web, que o seu criador, Tim Berners-Lee propôs: "O poder da Web está na sua universalidade. O acesso por todas as pessoas, não obstante a sua incapacidade, é um aspecto essencial." (Tim Berners-Lee - Diretor do W3C).

Como trabalhos futuros propõe-se o desenvolvimento de uma rede social acessível de acordo com os problemas relatados e a integração ao AVA Eduquito, desenvolvido pelo NIEE. ${ }^{2}$

\section{Agradecimento}

A participação do usuário cego evidenciou as dificuldades reais que esta parcela da população enfrenta no uso diário desta rede social, trazendo uma contribuição valiosa para esta a análise. Participação esta, que possibilitou-nos o exercício da empatia e que agradecemos afetuosamente.

\section{Referências}

CONFORTO, Débora; SANTAROSA, Lucila M. C. Acessibilidade à Web: Internet para Todos. Informática na Educação: Teoria, Prática - PGIE/UFRGS. V. 5 Nº 2 p. 87-102, nov/2002.

FACEBOOK. Disponível em: http://www.facebook.com. Acesso em: 09 out 2012.

BARROS, D. M. V. et al. (2011) Educação e tecnologias: reflexão, inovação e práticas. Lisboa: [s..n.]

MORO E. L. S. Ambientes Virtuais de Aprendizagem e Recursos da Web 2.0 em contexto hospitalar: rompendo a exclusão temporária de adolescentes com fibrose cística. $233 \mathrm{f}$. Tese (Doutorado em Informática na Educação) - Universidade Federal do Rio Grande do Sul. Centro Interdisciplinar de Novas Tecnologias. Programa de Pós Graduação em Informática na Educação, Porto Alegre. 2011.

PALLOFF, Rena M.; PRATT Keith. Construindo Comunidades de Aprendizagem no Ciberespaço estratégias eficientes para sala de aula on-line. Porto Alegre: Artmed, 2002.

RECUERO, Raquel. Redes Sociais na Internet. Porto Alegre: Sulina, 2008. 
SANTAROSA L. M. C. et al. Tecnologias Digitais Acessíveis. Porto Alegre: JSM Comunicação Ltda., 2010.

VIGOTSKY, L. S. A formação social da mente: o desenvolvimento dos processos psicológicos superiores. 7. ed. São Paulo: Martins Fontes, 2007.

.; LURIA, A. R.; LEONTIEV, A. N. Linguagem, desenvolvimento e aprendizagem. São Paulo: Ícone, 2006.

W3C. Introduction to Understanding WCAG 2.0. Disponível em: <http://www.w3.org/TR/UNDERSTANDING-WCAG20/intro.html> Acesso em: ago 2012.

Submetido em 15 de agosto de 2013.

Aprovado em 04 de setembro de 2013.

Rosana Wagner: Universidade Federal do Rio Grande do Sul - Programa de Pós-Graduação em Informática na Educação. Porto Alegre-RS - Brasil.

E-mail: rosanawagner@gmail.com

Sandra Piovesan: Universidade Federal do Rio Grande do Sul - Programa de Pós-Graduação em Informática na Educação. Porto Alegre-RS - Brasil.

E-mail: sanpiovesan@gmail.com

Lisete Rodrigues: Universidade Federal do Rio Grande do Sul - Programa de Pós-Graduação em Educação. Porto Alegre-RS - Brasil.

E-mail: liseteporto@gmail.com 